Recepción: 20 / 04 / 2017

Aceptación: 20 / 05 / 2017

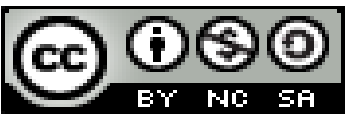

Ciencias de la Salud

Publicación: 15 / 07 / 2017

Artículo Científico

\title{
Desnutrición proteica y calórica en los adultos mayores
}

\author{
Protein and calorie malnutrition in the elderly
}

\section{desnutrição protéico-calórica em adultos mais velhos}

Jaramillo Zuñiga-Elizabeth I

unicornio1206@outlook.es

Martha A. Ayala-Paredes II

aliciadevillagran@hotmail.com

Nancy J. Rodríguez-Calderón III

nancyrodriguezcalderon@hotmail.com

\author{
Adriana M. Intriago-Rosado IV \\ aintriago54@hotmail.com \\ Sonia B. Gómez-Vergara V \\ sonygomez28@hotmail.com
}

Correspondencia: unicornio1206@ outlook.es

\footnotetext{
Magister en Nutrición; Licenciada en Enfermería; Universidad de Guayaquil, Guayaquil, Ecuador. Diplomado en Docencia Superior; Magister en Gerencia Educativa; Especialista en Gestión de Procesos Educativos; Psicóloga Industrial; Universidad de Guayaquil, Guayaquil, Ecuador.

Licenciada en Terapia de Lenguaje; Universidad de Guayaquil, Guayaquil, Ecuador.

Diplomado en Docencia Superior; Obstetriz; Universidad de Guayaquil, Guayaquil, Ecuador.

Magister en Nutrición; Diplomado en Docencia Superior; Obstetriz; Universidad de Guayaquil, Guayaquil, Ecuador.
} 


\section{Resumen}

El estado de desnutrición de los adultos mayores se presenta como resultado de una ingesta insuficiente de los nutrientes esenciales para el organismo. La investigación se la realizó en el centro de cuidados Hogar del Corazón de Jesús de la ciudad Guayaquil, Ecuador. Tuvo como objetivo: Establecer el grado de desnutrición de los adultos mayores de acuerdo a los niveles proteicos. Metodología: Investigación cualitativa, el método usado fue el estudio de casos. Como instrumentos para recolectar datos se utilizaron: "Mini Nutricional Assessment" y la ficha de registro de datos del adulto mayor. Resultados: Existe una prevalencia del 10\% de desnutrición, 50\% tiene un estado nutricional normal y $40 \%$ tiene riesgo de desnutrición. Existe una prevalencia de desnutrición en los adultos mayores a 76 años o más del sexo femenino. Los grados de desnutrición según niveles de albúmina sérica fueron: $10 \%$ desnutrición leve, $28 \%$ desnutrición moderada y 10\% desnutrición severa.

Palabras Claves: Ingesta; albúmina; nutrientes; proteicos; malnutrición 


\section{Summary}

The malnutrition status of the elderly occurs as a result of insufficient intake of essential nutrients for the body. The research was carried out at the Hogar de Corazón de Jesús care center in the city of Guayaquil, Ecuador. It aimed to: Establish the degree of malnutrition of older adults according to protein levels. Methodology: Qualitative research, the method used was the case study. As instruments to collect data were used: "Mini Nutritional Assessment" and the data record of the elderly. Results: There is a prevalence of $10 \%$ malnutrition, $50 \%$ have a normal nutritional status and $40 \%$ are at risk of malnutrition. There is a prevalence of malnutrition in the elderly to 76 years or more of the female sex. The levels of malnutrition according to serum albumin levels were: $10 \%$ mild malnutrition, $28 \%$ moderate malnutrition and $10 \%$ severe malnutrition.

Key Words: Ingestion; albumin; nutrients; proteins; malnutrition. 


\section{Resumo}

O estado de desnutrição dos idosos ocorre como resultado da ingestão inadequada de nutrientes essenciais para o corpo. A pesquisa foi feita no Centro de Atendimento ao Início do Sagrado Coração da cidade de Guayaquil, Equador. Ele destina-se a: Definir o grau de desnutrição entre adultos mais velhos de acordo com os níveis de proteína. Metodologia: Pesquisa qualitativa, o método utilizado foi o estudo de caso. Como foram utilizados instrumentos para coleta de dados: "Mini Avaliação Nutricional" e folha de registro de dados dos idosos. Resultados: Há uma prevalência de $10 \%$ de desnutrição, $50 \%$ têm um estado nutricional normal e $40 \%$ em risco de desnutrição. Há uma prevalência de desnutrição em idosos de 76 anos ou mais fêmeas adultas. Graus de desnutrição como os níveis de albumina de soro foram de $10 \%$ desnutrição leve, $28 \%$ desnutrição moderada e $10 \%$ subnutrição grave.

Palavras-chave: Ingest; albumina; nutrientes; proteínas; subnutrição 


\section{Introduccion.}

La esperanza de vida ha crecido en el siglo XX y la tendencia demográfica ha favorecido al adulto mayor. La (OMS, 2016) define la malnutrición como: “carencias, excesos o desequilibrios en la ingesta de energía, proteínas y/o otros nutrientes". (p. 78). Tanto el exceso como el déficit implican una alteración del metabolismo y un mal uso de los nutrientes en el cuerpohumano.

“Entre el año 2000 y el 2050, el número de personas del planeta mayores de 60 años de edad se duplicará del $11 \%$ al 22\%, lo que equivale que este grupo de personas aumentará de 605 millones a 2000 millones en el lapso de medio siglo" (p. 2)

Morley (2015) afirma que, en caso de existir un déficit proteico-calórico esto ocasionará en el organismo padecimientos preponderantes de desnutrición por múltiples causas tales como: enfermedades crónicas y agudas, factores socioeconómicos (bajo nivel de ingresos y de instrucción educativa), problemas dentarios y de deglución, infecciones gastrointestinales, traumatismos, medicamentos (que afectan la ingesta, la absorción y utilización de los nutrientes), trastornos mentales, ansiedad, depresión, entre otros, conllevando al deterioro general de la calidad de vida.

El aumento de adultos mayores aumenta ya que el promedio de vida se va extendiendo gracias a los avances de la ciencia, aumentando el interés por su salud y las condiciones de una nutrición adecuada.

(INEC, 2010) informa: "La población adulta mayor (más de 65 años) llega, en Ecuador, a 940 mil personas; anualmente fallecen alrededor de 34 mil ecuatorianos" Algunos residen en hogares de cuidado especializados para la estadía de ellos, contando estos centros en muchos casos con especialistas geriatras altamente calificados. La edad de los adultos mayores de estos hogares de 
cuidados fluctúa entre 60 y 90 años aproximadamente y muchos tienen desnutrición, por lo cual es indispensable tomar medidas para proveerles de una adecuada alimentación.

El estudio realizado representa un gran aporte a la comunidad investigativa desarrollado en el "Hogar Corazón de Jesús" de la ciudad de Guayaquil, en el que reside una población de adultos mayores significativa, para los que en el análisis previo de la investigación se logró evidenciar el requerimiento en la identificación del estado nutricional, estableciendo como necesario un seguimiento posterior, con la finalidad de hacer las correcciones nutricionales correspondientes.

\section{Materiales y métodos (Metodología)}

Los instrumentos que se utilizaron para recolectar los datos fueron:

- Ficha de registro de datos del adulto mayor para recolectar los datos demográficos (edad, sexo y nivel de instrucción), los valores de albúmina sérica, presencia de enfermedades crónicas, datos antropométricos (talla y peso) de los adultos mayores.

- Se utilizó el MNA (Mini Nutritional Assessment) para valorar el estado nutricional de los adultos mayores del centro de cuidados Hogar del Corazón de Jesús, debido a que es instrumento económico, fácil de utilizar y no requiere de mucho tiempo para su realización.

La presente investigación utilizó el método estudio de casos para analizar la desnutrición en los adultos mayores. Se la realizó durante los meses de agosto, septiembre octubre en el centro de cuidados para adultos mayores en el Hogar del Corazón de Jesús de la Junta de Beneficencia de Guayaquil, que está ubicado en la ciudad de Guayaquil, Av. Pedro Menéndez Gilbert (junto a la sala de velación del Cementerio Patrimonial). El estudio analizó las categorías: Estado nutricional del adulto mayor factores demográficos y grado de desnutrición de acuerdo a los niveles proteicos. 
La unidad de análisis que constituye el objeto de la presente investigación son los adultos mayores del centro.

\section{Resultados}

Los resultados de la aplicación del MNA en su segunda fase (evaluación) muestran que del total de adultos mayores evaluados el 50\% tenía un estado nutricional normal, el 40\% tenía riesgo de desnutrición y el 10\% tenía desnutrición.

\section{Discusión}

- Según los resultados de la presente investigación existe una prevalencia de desnutrición en: el sexo femenino; en los adultos mayores que tenían 76 años o más, con instrucción primaria. Se determinó el grado de desnutrición de los adultos mayores de acuerdo a los niveles de la albúmina sérica los resultados fueron: $10 \%$ tenía desnutrición leve, $28 \%$ tenía desnutrición moderada y $10 \%$ tenía desnutrición severa.

- La presente investigación se correlaciona con otra realizada por Andrade (2011) autora de la tesis: "Nivel de Depresión en Adultos Mayores de 65 Años y su Impacto en el Estado Nutricional en la Parroquia Asunción. Cantón Girón. Azuay. 2011”, que realizó la valoración nutricional de adultos mayores utilizando como instrumento el MNA (Mini Nutritional Assessment) y encontró que el $84,4 \%$ de los adultos mayores tenían riesgo de desnutrición y desnutrición. En cambio el presente estudio halló un 50\% con riesgo de desnutrición y desnutrición, lo que deja ver que el MNA es una herramienta que ayuda a identificar casos de desnutrición y riesgo de desnutrición en la población de adultos mayores.

- $\quad$ En otra investigación realizada por Palacios (2012) (bajo el título: "Evaluación del estado 
nutricional mediante el MNA (Mini Nutritional Assessment) en relación a factores sociales y demográficos en adultos mayores no institucionalizados de la parroquia Rumipamba, Provincia de Tungurahua 2011", se determinó que el estado del adulto mayor está muy relacionado con la edad, puesto que a medida que cuando la edad es riesgo de desnutrición es mayor. Lo anterior coincide con los resultados encontrados en la presente investigación, puesto que existe una prevalencia de desnutrición en los ancianos que tenían más edad (76 o más años), mientras que en los que tenían menos edad no había ningún caso de desnutrición.

- $\quad$ La investigación de Espinosa (2014) (7) "Prevalencia y factores asociados a malnutrición en adultos mayores hospitalizados en el departamento de clínica del Hospital Vicente Corral Moscoso, 2013” determinó que existía mayor presencia de desnutrición en personas mayores a 75 años, sexo femenino y estado civil viudo. Como factores de riesgo se identificó el no uso de prótesis dental y a la dificultad para alimentarse. Los resultados anteriores son similares a los encontrados en la presente investigación, puesto que se determinó que existía una prevalencia de desnutrición en los ancianos que tenían más edad (76 o más años) y en los ancianos de sexo femenino.

- La investigación de Hilerio (2009) (9) "Estado nutricional en el adulto mayor institucionalizado" evaluó adultos mayores de 4 asilos del estado de Colima Según esta investigación existe una tendencia de los adultos mayores hacia la desnutrición (55\% se encontraban en riesgo de desnutrición). Los resultados anteriores son casi similares a los del presente estudio puesto que el $40 \%$ de adultos mayores tenía riesgo de desnutrición y el $10 \%$ tenía desnutrición. 


\section{Conclusiones.}

- $\quad$ El presente estudio no abordó la prevalencia de la desnutrición de los adultos mayores según el estado civil que tenían. Determinó el grado de desnutrición considerando solamente los niveles de albúmina sérica ya que eran los únicos datos de laboratorio actualizados que había en la historia clínica de los adultos mayores evaluados.

- $\quad$ Se pueden realizar futuras investigaciones que determinen el grado de desnutrición considerando además de la albúmina sérica, los niveles de colesterol y de linfocitos que son otros indicadores que permiten establecer el grado de desnutrición. A diferencia de los referentes empíricos revisados, el presente estudio identificó la prevalencia de la desnutrición de acuerdo al nivel de instrucción.

\section{Bibliografía}

OMS. (2016, diciembre 12). Envejecimiento y ciclo de vida. Retrieved from http://www.who.int/ageing/about/facts/es/

Morley, J. (2015, diciembre 9). Desnutrición calórico-proteica. Retrieved from http://www.msdmanuals.com/es-ec/professional/trastornosnutricionales/desnutrici\%C3\%B3n/desnutrici\%C3\%B3n-cal\%C3\%B3rico-proteica

INEC. (2010). Las condiciones de vida de los ecuatorianos. Recuperado el 25 de febrero de 2016, de http://www.ecuadorencifras.gob.ec/documentos/web-

inec/ECV/Publicaciones/ECV_Folleto_de_ind_sociales.pdf

Gutiérrez, J., Serralde, A., \& Guevara, M. (2007). Prevalencia de desnutrición del adulto mayor al ingreso hospitalario. Nutrición Hospitalaria.

Andrade, C. (2011). Nivel de Depresión en Adultos mayores. Riobamba: Escuela Superior Politécnica de Chimborazo. 
Palacios, S. (2012). Evaluación del estado nutricional. Riobamba: Escuela Politécnica de Chimborazo.

Espinosa, H. (2014). Prevalencia y factores asociados a malnutrición en adultos mayores. Cuenca: Universidad de Cuenca.

Gallo, A., Núñez, M., Reyes, M., \& Valdez, E. (s/f.). Guía alimentaria para adultos mayores. México: Universidad Mundial.

Hilerio, A. (2009). Estado nutricional en el adulto mayor institucionalizado. Colima: Universidad de Colima. 